FACTA UNIVERSITATIS (NIŠ)

Ser. Math. Inform. Vol. 36, No 1 (2021), 65-77

https://doi.org/10.22190/FUMI200309006C

Original Scientific Paper

\title{
TRIANGULAR $A$-STATISTICAL RELATIVE UNIFORM CONVERGENCE FOR DOUBLE SEQUENCES OF POSITIVE LINEAR OPERATORS
}

\author{
Selin Çınar \\ Faculty of Science and Arts, Department of Mathematics, \\ Sinop University, 57000 Sinop, Turkey
}

\begin{abstract}
In this paper, we introduce the concept of triangular $A$-statistical relative convergence for double sequences of functions defined on a compact subset of the real two-dimensional space. Based upon this new convergence method, we prove Korovkintype approximation theorem. Finally, we give some further developments.

Keywords: positive linear operators, the double sequences, regular matrix, triangular A-statistical convergence, Korovkin theorem.
\end{abstract}

\section{Introduction}

Classical Bohman-Korovkin theorem is a well known theorem which has an important place in approximation theory $([13],[16],[21])$. This theorem establishes the uniform convergence in the space $C[a, b]$ of all continuous real functions defined on the interval $[a, b]$, for a sequence of positive linear operators $\left(L_{n}\right)$, assuming the convergence by the test functions $f_{r}(s)=s^{r}, r=0,1,2$. Moreover, different finite classes of test functions were studied, in both one and multi-dimensional case. Many mathematicians studied and improved this theory by defining positive linear operators via convergence methods on various function spaces ([1], [4], [5], [6], [15], [18], [20], [23], [24], [25], [26], [34]). In recent years, general versions of Korovkin theorem have been studied, in which a more general notion of convergence is used. One of these convergences is the statistical convergence first introduced by Fast and Steinhaus ([17], [30]). Korovkin type approximation theorems have been first studied via the notion of statistical convergence by Gadjiev and Orhan [19]. For

Received March 9, 2020; accepted May 30, 2020.

Corresponding Author: Selin Çınar, Faculty of Science and Arts, Department of Mathematics, Sinop University, 57000 Sinop, Turkey | E-mail: scinar@sinop.edu.tr 2010 Mathematics Subject Classification. 40A35; 41A25, 41A36

(C) 2021 by University of Niš, Serbia | Creative Commons License: CC BY-NC-ND 
double sequences of positive linear operators, statistical convergence and some of its generalizations to convergence generated by summability matrix methods were carried on by Demirci and Dirik ([8], [14]). With the help of these studies, triangular $A$-statistical convergence which is a different kind of statistical convergence was identified by Bardaro et. al. ([2], [3]).

Recently, Demirci and Orhan [11] have defined the statistically relatively uniform convergence by using statistical convergence and the relatively uniform convergence and established its use in the Korovkin-type approximation theory. Also, a type of modular convergence, called relative modular convergence, was introduced in [33] originated by studies in modular spaces and these studies continued ([9], [10], [12]).

Our main aim in this paper is to present a new kind of statistical convergence for double sequence, called triangular $A$-statistical relative uniform convergence. We will compare this new convergence with triangular $A$-statistical convergence and obtain more general results.

Now, we begin with the definitions and notations required for this article.

E. H. Moore [22] introduced the notion of uniform convergence of a sequence of functions relative to a scale function. Then, E. W. Chittenden [7] gave the following definition of relatively uniform converge which is equivalent to the definition given by Moore:

A sequence $\left(f_{n}\right)$ of functions, defined on any compact subset of real space, converges relatively uniformly to a limit function $f$ if there exists a function $\sigma(s)$, such that for every $\varepsilon>0$ there exists an integer $n_{\varepsilon}$ such that for every $n>n_{\varepsilon}$ the inequality

$$
\left|f_{n}(s)-f(s)\right|<\varepsilon|\sigma(s)|
$$

holds uniformly in $s$. The sequence $\left(f_{n}\right)$ is said to converge uniformly relatively to the scale function $\sigma$ or more briefly relatively uniformly. Similarly, Dirik and Şahin [31] gave the following for double sequences of functions:

A double sequence $\left(f_{i, j}\right)$ of functions, defined on any compact subset of the real two-dimensional space, converges relatively uniformly to a limit function $f$ if there exists a function $\sigma(s, t)$, called a scale function such that for every $\varepsilon>0$ there is an integer $n_{\varepsilon}$ such that for every $i, j>n_{\varepsilon}$ the inequality

$$
\left|f_{i, j}(s, t)-f(s, t)\right|<\varepsilon|\sigma(s, t)|
$$

holds uniformly in $(s, t)$. The double sequence $\left(f_{i, j}\right)$ is said to converge uniformly relatively to scale function $\sigma$ or more briefly, relatively uniformly.

Let $A=\left(a_{i, j}\right)$ be a two-dimensional matrix transformation. For a double sequence $x=\left(x_{i, j}\right)$ of real numbers, we put

$$
(A x)_{i}:=\sum_{j=1}^{\infty} a_{i, j} x_{i, j},
$$


if the series is convergent. We will say that $A$ is regular if $\lim A x=L$ whenever $\lim x=L$. The well-established characterization for regular two-dimensional matrix transformation is known as the Silverman-Toeplitz conditions [32]:

(i) $\|A\|=\sup _{i \in \mathbb{N}} \sum_{j=1}^{\infty}\left|a_{i, j}\right|<\infty$

(ii) $\lim _{i} a_{i, j}=0$ for each $j \in \mathbb{N}$,

(iii) $\lim _{i} \sum_{j=1}^{\infty} a_{i, j}=1$.

A double sequence $x=\left(x_{i, j}\right)$ of real numbers, $i, j \in \mathbb{N}$, the set of all positive integers, is said to be convergent in the Pringsheim's sense or $P$-convergent if for each $\varepsilon>0$ there exists $N \in \mathbb{N}$ such that $\left|x_{i, j}-L\right|<\varepsilon$ whenever $i, j>N$ and $L$ is called the Pringsheim limit ( $\operatorname{denoted}$ by $P-\lim _{i, j} x_{i, j}=L$ ) [28]. More briefly, we will say that such an $x$ is $P$-convergent to $L$. A double sequence is said to be bounded if there exists a positive number $K$ such that $\left|x_{i, j}\right| \leq K$ for all $(i, j) \in \mathbb{N}^{2}=\mathbb{N} \times \mathbb{N}$. Note that in contrast to the case for single sequences, a convergent double sequences need not to be bounded, provided the double sequences converges in Pringsheim's sense for every $(i, j) \in \mathbb{N}^{2}$.

Let now $A=\left(a_{n, m, i, j}\right)$ be a four-dimensional matrix and $x=\left(x_{i, j}\right)$ be a double sequence. Then the double (transformed) sequence, $A x:=\left((A x)_{n, m}\right)$, is denoted by

$$
(A x)_{n, m}=\sum_{i, j=1,1}^{\infty, \infty} a_{n, m, i, j} x_{i, j}
$$

where it is assumed that the summation exists as a Pringsheim limit for each $(n, m) \in \mathbb{N}^{2}$.

Recall that four-dimensional matrix $A=\left(a_{n, m, i, j}\right)$ is said to be $R H$-regular if it maps every bounded $P$-convergent sequence into a $P$-convergent sequence with the same $P$-limit. The Robison-Hamilton conditions (see also [29]) state that a four-dimensional matrix $A=\left(a_{n, m, i, j}\right)$ is $R H$-regular if and only if

(i) $\quad P-\lim _{n, m} a_{n, m, i, j}=0$ for each $i$ and $j$

(ii) $P-\lim _{n, m} \sum_{i, j}^{\infty, \infty} a_{n, m, i, j}=1$,

(iii) $P-\lim _{n, m} \sum_{i=1}^{\infty}\left|a_{n, m, i, j}\right|=0$ for each $j \in \mathbb{N}$,

(iv) $P-\lim _{n, m} \sum_{j=1}^{\infty}\left|a_{n, m, i, j}\right|=0$ for each $i \in \mathbb{N}$,

(v) $\sum_{i, j=1,1}^{\infty, \infty}\left|a_{n, m, i, j}\right|$ is $P$-convergent for every $(n, m) \in \mathbb{N}^{2}$, 
(vi) there exist finite positive integers $A$ and $B$ such that

$$
\sum_{i, j>B}\left|a_{n, m, i, j}\right|<A
$$

for every $(n, m) \in \mathbb{N}^{2}$.

Let $A=\left(a_{n, m, i, j}\right)$ be a nonnegative $R H$-regular summability matrix. If $K \subset$ $\mathbb{N}^{2}$, then the $A$-density of $K$ is denoted by

$$
\delta_{A}^{2}(K):=P-\lim _{n, m} \sum_{(i, j) \in K} a_{n, m, i, j}
$$

provided that the limit on the right-hand side exists in the Pringsheim sense. A real double sequence $x=\left(x_{i, j}\right)$ is said to be $A$-statistically convergent to $L$ and denoted by $s t_{A-}^{2} \lim _{i, j} x_{i, j}=L$ if, for every $\varepsilon>0$,

$$
P-\lim _{n, m} \sum_{(i, j) \in K(\varepsilon)} a_{n, m, i, j}=0,
$$

where $K(\varepsilon)=\left\{(i, j) \in \mathbb{N}^{2}:\left|x_{i, j}-L\right| \geq \varepsilon\right\}$. If we take $A=C(1,1)$, then $C(1,1)-$ -statistical convergence coincides with the notion of statistical convergence for double sequences $([27])$, where $C(1,1)=\left(c_{i, j, n, m}\right)$ is the double Cesàro matrix, defined by $c_{i, j, n, m}=1 / i j$ if $1 \leq n \leq i, 1 \leq m \leq j$ and $c_{i, j, n, m}=0$ otherwise. We state the set of all $A$-statistically convergent double sequences by $s t_{A}^{2}$.

\section{Triangular $A$ - Statistical Relative Uniform Convergence}

First, we recall some definitions given in [2].

Let $A=\left(a_{i, j}\right)$ be a nonnegative regular summability matrix, $K \subset \mathbb{N}^{2}$ be a nonempty set, and for every $i \in \mathbb{N}$, let $K_{i}:=\{j \in \mathbb{N}:(i, j) \in K, j \leq i\}$. Triangular $A$-density of $K$, is given by

$$
\delta_{A}^{T}(K):=\lim _{i} \sum_{j \in K_{i}} a_{i, j},
$$

provided that the limit on right-hand side exists in $\mathbb{R}$.

In a similar manner to the natural density, we can give some properties for the triangular $A$-density:

i) if $K_{1} \subset K_{2}$, then $\delta_{A}^{T}\left(K_{1}\right) \subset \delta_{A}^{T}\left(K_{2}\right)$,

ii) if $K$ has triangular $A$-density, then $\delta_{A}^{T}\left(\mathbb{N}^{2} \backslash K\right)=1-\delta_{A}^{T}(K)$.

Let $A=\left(a_{i, j}\right)$ be a nonnegative regular summability matrix. The double sequence $x=\left(x_{i, j}\right)$ is triangular $A$-statistically convergent to $L$ provided that for every $\varepsilon>0$

$$
\lim _{i} \sum_{j \in K_{i}(\varepsilon)} a_{i, j}=0
$$


where $K_{i}(\varepsilon)=\left\{j \in \mathbb{N}: j \leq i,\left|x_{i, j}-L\right| \geq \varepsilon\right\}$ and this denoted by $s t_{A}^{T}-\lim _{i} x_{i, j}=$ $L$. We should note that if we take $A=C_{1}:=\left(c_{i, j}\right)$, the Cesàro matrix defined by

$$
c_{i, j}:= \begin{cases}\frac{1}{i}, & \text { if } 1 \leq j \leq i \\ 0, & \text { otherwise }\end{cases}
$$

then the triangular $A$-density is called triangular density which is denoted by

$$
\delta^{T}(K)=\lim _{i} \frac{1}{i}\left|K_{i}\right|
$$

where $\left|K_{i}\right|$ be the cardinality of $K_{i}$. According to the above definitions triangular $A$-statistical convergent reduces triangular statistical convergent.

Let $S$ is a compact subset of the real two-dimensional space. By $C(S)$ we define the space of all continuous real valued functions on $S$ and $\|f\|_{C(S)}$ denotes the usual supremum norm of $f$ in $C(S)$. Let $f$ and $f_{i, j}$ belong to $C(S)$.

Definition 2.1. Let $A=\left(a_{i, j}\right)$ be a nonnegative regular summability matrix. A double sequence of fuctions $\left(f_{i, j}\right)$ is said to triangular $A$-statistically uniformly convergent to $f$ on $S$ provided that for every $\varepsilon>0$,

$$
\lim _{i} \sum_{j \in K_{i}(\varepsilon)} a_{i, j}=0
$$

where $K_{i}(\varepsilon)=\left\{j \in \mathbb{N}: j \leq i, \sup _{(s, t) \in S}\left|f_{i, j}(s, t)-f(s, t)\right| \geq \varepsilon\right\}$. In this case, we write $f_{i, j} \rightrightarrows f\left(s t_{A}^{T}\right)$.

Definition 2.2. Let $A=\left(a_{i, j}\right)$ be a nonnegative regular summability matrix. $\left(f_{i, j}\right)$ is said to be triangular $A$-statistically relatively uniformly convergent to $f$ on $S$ if there exists a function $\sigma(s, t),|\sigma(s, t)|>0$, called a scale function, provided that for every $\varepsilon>0$,

$$
\lim _{i} \sum_{j \in K_{i}(\varepsilon)} a_{i, j}=0
$$

where $K_{i}(\varepsilon)=\left\{j \in \mathbb{N}: j \leq i, \sup _{(s, t) \in S}\left|\frac{f_{i, j}(s, t)-f(s, t)}{\sigma(s, t)}\right| \geq \varepsilon\right\}$. In this case, we write $f_{i, j} \rightrightarrows f\left(s t_{A}^{T}, \sigma\right)$.

It will be observed that triangular $A$-statistical uniform convergence is the special case of triangular $A$-statistical relative uniform convergence in which the scale function is a non-zero constant.

Example 2.1. Take $A=C_{1}$ and $S=[0,1] \times[0,1]$. For each $(i, j) \in \mathbb{N}^{2}$, define $\gamma_{i, j}: S \rightarrow$ $\mathbb{R}$ by

$$
\gamma_{i, j}(s, t)= \begin{cases}\frac{2 i^{2} j^{2} s t}{1+i^{3} j^{3} s^{2} t^{2}}, & i \text { and } j \text { are square } \\ 0, & \text { otherwise }\end{cases}
$$


Since $\left\|\gamma_{i, j}-\gamma\right\|_{C(S)}=1$, this sequence does not triangular statistically uniform convergent to $\gamma=0$, but triangular statistically relatively uniform convergent to $f=0$, with a scale function defined by,

$$
\sigma(s, t)= \begin{cases}\frac{1}{s t}, & \text { if }(s, t) \in(0,1] \times(0,1] \\ 0, & \text { if } s=0 \text { or } t=0\end{cases}
$$

clearly, for every $\varepsilon>0$,

$$
\lim _{i} \frac{1}{i}\left|\left\{j \in \mathbb{N}: j \leq i, \sup _{(s, t) \in S}\left|\frac{\gamma_{i, j}(s, t)-\gamma(s, t)}{\sigma(s, t)}\right| \geq \varepsilon\right\}\right|=0 .
$$

\section{A Korovkin-type approximation theorem}

Let $L$ be a linear operator from $C(S)$ into itself and is called positive, if $L(f) \geq 0$, for all $f \geq 0$. Also, we denote the value of $L(f)$ at a point $(s, t) \in S$ by $L(f(u, v) ; s, t)$ or, briefly, $L(f ; s, t)$.

Theorem 3.1. [2] Let $A=\left(a_{i, j}\right)$ be a nonnegative regular summability matrix and $\left(L_{i, j}\right)$ be a double sequence of positive linear operators from $C(S)$ into $C(S)$. Then for every $f \in C(S)$ we have

$$
s t_{A}^{T}-\lim _{i}\left\|L_{i, j}(f)-f\right\|_{C(S)}=0
$$

if and only if

$$
s t_{A}^{T}-\lim _{i}\left\|L_{i, j}\left(f_{r}\right)-f_{r}\right\|_{C(S)}=0 \text { for every } r=0,1,2,3,
$$

where $f_{0}(s, t)=1, f_{1}(s, t)=s, f_{2}(s, t)=t, f_{3}(s, t)=s^{2}+t^{2}$.

Now we have the following Korovkin type approximation theorem for triangular $A$-statistical relative convergence that is our main theorem.

Theorem 3.2. Let $A=\left(a_{i, j}\right)$ be a nonnegative regular summability matrix. Let $\left(L_{i, j}\right)$ be a double sequence of positive linear operators from $C(S)$ into $C(S)$. Then, for all $f \in C(S)$ we have

$$
L_{i, j}(f) \rightrightarrows f \quad\left(s t_{A}^{T}, \sigma\right)
$$

if and only if,

$$
L_{i, j}\left(f_{r}\right) \rightrightarrows f_{r} \quad\left(s t_{A}^{T}, \sigma_{r}\right) \quad(r=0,1,2,3)
$$

where $f_{0}(s, t)=1, f_{1}(s, t)=s, f_{2}(s, t)=t, f_{3}(s, t)=s^{2}+t^{2}$ and $\sigma_{r}(s, t)=$ $\max \left\{\left|\sigma_{r}(s, t)\right| ; r=0,1,2,3\right\},\left|\sigma_{r}(s, t)\right|>0$ and $\sigma_{r}(s, t)$ is unbounded, $r=0,1,2,3$. 
Proof. Since each $f_{r} \in C(S)(r=0,1,2,3)$, (3.3) $\Longrightarrow(3.4)$ is obvious. Suppose now that (3.4) holds. By continuity of $f$ on the compact set $S$, we can write $|f(s, t)| \leq M$ where $M:=\|f\|_{C(S)}$. Also, since $f$ is continuous on $S$, for every $\varepsilon>0$, there exists $\delta>0$ such that $|f(u, v)-f(s, t)|<\varepsilon$ for all $(u, v) \in S$ satisfying $|u-s|<\delta$ and $|v-t|<\delta$. Hence, we get

$$
|f(u, v)-f(s, t)|<\varepsilon+\frac{2 M}{\delta^{2}}\left\{(u-s)^{2}+(v-t)^{2}\right\}
$$

Since $L_{i, j}$ is linear and positive, we obtain

$$
\begin{aligned}
& \left|L_{i, j}(f ; s, t)-f(s, t)\right|=\mid L_{i, j}(f(u, v)-f(s, t) ; s, t) \\
& -f(s, t)\left(L_{i, j}\left(f_{0} ; s, t\right)-f_{0}(s, t)\right) \\
& \leq\left|L_{i, j}\left(\varepsilon+\frac{2 M}{\delta^{2}}\left\{(u-s)^{2}+(v-t)^{2}\right\} ; s, t\right)\right| \\
& +M\left|L_{i, j}\left(f_{0} ; s, t\right)-f_{0}(s, t)\right| \\
& \leq\left(\varepsilon+M+\frac{2 M}{\delta^{2}}\left(A^{2}+B^{2}\right)\right)\left|L_{i, j}\left(f_{0} ; s, t\right)-f_{0}(s, t)\right| \\
& +\frac{4 M}{\delta^{2}} A\left|L_{i, j}\left(f_{1} ; s, t\right)-f_{1}(s, t)\right| \\
& +\frac{4 M}{\delta^{2}} B\left|L_{i, j}\left(f_{2} ; s, t\right)-f_{2}(s, t)\right| \\
& +\frac{2 M}{\delta^{2}}\left|L_{i, j}\left(f_{3} ; s, t\right)-f_{3}(s, t)\right|+\varepsilon
\end{aligned}
$$

where $A:=\max |s|, B:=\max |t|$. Now we multiply the both-sides of the above inequality by $\frac{1}{|\sigma(s, t)|}$,

$$
\begin{gathered}
\left|\frac{L_{i, j}(f ; s, t)-f(s, t)}{\sigma(s, t)}\right| \leq K\left\{\left|\frac{L_{i, j}\left(f_{0} ; s, t\right)-f_{0}(s, t)}{\sigma(s, t)}\right|+\left|\frac{L_{i, j}\left(f_{1} ; s, t\right)-f_{1}(s, t)}{\sigma(s, t)}\right|\right. \\
\left.(3.6)\left|\frac{L_{i, j}\left(f_{2} ; s, t\right)-f_{2}(s, t)}{\sigma(s, t)}\right|+\left|\frac{L_{i, j}\left(f_{3} ; s, t\right)-f_{3}(s, t)}{\sigma(s, t)}\right|\right\}+\frac{\varepsilon}{|\sigma(s, t)|},
\end{gathered}
$$

where $K=\max \left\{\varepsilon+M+\frac{2 M}{\delta^{2}}\left(A^{2}+B^{2}\right), \frac{4 M}{\delta^{2}} A, \frac{4 M}{\delta^{2}} B, \frac{2 M}{\delta^{2}}\right\}$ and where $\sigma(s, t)=\max \left\{\left|\sigma_{r}(s, t)\right| ; r=0,1,2,3\right\}$. Taking the supremum over $(s, t) \in S$, we get

$$
\begin{aligned}
\sup _{(s, t) \in S}\left|\frac{L_{i, j}(f ; s, t)-f(s, t)}{\sigma(s, t)}\right| \leq & \sup _{(s, t) \in S} \frac{\varepsilon}{|\sigma(s, t)|}+K\left\{\sup _{(s, t) \in S}\left|\frac{L_{i, j}\left(f_{0} ; s, t\right)-f_{0}(s, t)}{\sigma_{0}(s, t)}\right|\right. \\
& +\sup _{(s, t) \in S}\left|\frac{L_{i, j}\left(f_{1} ; s, t\right)-f_{1}(s, t)}{\sigma_{1}(s, t)}\right| \\
& +\sup _{(s, t) \in S}\left|\frac{L_{i, j}\left(f_{2} ; s, t\right)-f_{2}(s, t)}{\sigma_{2}(s, t)}\right| \\
& \left.+\sup _{(s, t) \in S}\left|\frac{L_{i, j}\left(f_{3} ; s, t\right)-f_{3}(s, t)}{\sigma_{3}(s, t)}\right|\right\} .
\end{aligned}
$$


Now, for a given $r>0$, choose $\varepsilon>0$ such that $\sup _{(s, t) \in S} \frac{\varepsilon}{|\sigma(s, t)|}<r$. Then, setting $D_{i}:=\left\{j \in \mathbb{N}: j \leq i,\left\|\frac{L_{i, j}(f)-f}{\sigma}\right\|_{C(S)} \geq r\right\}$

$D_{i}^{r}:=\left\{j \in \mathbb{N}: j \leq i,\left\|\frac{L_{i, j}\left(f_{r}\right)-f r}{\sigma_{r}}\right\|_{C(S)} \geq \frac{r-\sup _{(s, t) \in S} \frac{\varepsilon}{|\sigma(s, t)|}}{4 K}\right\}, r=0,1,2,3$. It is easy to see that

$$
D_{i} \subseteq \cup_{r=0}^{3} D_{i}^{r}
$$

which gives, for all $i \in \mathbb{N}$, then

$$
\sum_{j \in D_{i}} a_{i, j} \leq \sum_{r=0}^{3} \sum_{j \in D_{i}^{r}} a_{i, j}
$$

Letting $i \rightarrow \infty$ and using (3.4), we obtain (3.5). The proof is complete.

If one replaces the scale function by a non-zero constant, then the Theorem 3.2 reduces to the Theorem 3.1 .

We now show that our result Theorem 3.2 is stronger than Theorem 3.1.

Example 3.1. Let consider the following Bernstein operators given by

$$
B_{i, j}(f ; s, t)=\sum_{k=0}^{i} \sum_{p=0}^{j} f\left(\frac{k}{i}, \frac{p}{j}\right)\left(\begin{array}{c}
i \\
k
\end{array}\right)\left(\begin{array}{c}
j \\
p
\end{array}\right) s^{k}(1-s)^{i-k} t^{p}(1-t)^{j-p}
$$

where $(s, t) \in S=[0,1] \times[0,1] ; f \in C(S)$. Also, observe that

$$
\begin{aligned}
B_{i, j}\left(f_{0} ; s, t\right) & =f_{0}(s, t), \\
B_{i, j}\left(f_{1} ; s, t\right) & =f_{1}(s, t), \\
B_{i, j}\left(f_{2} ; s, t\right) & =f_{2}(s, t), \\
B_{i, j}\left(f_{3} ; s, t\right) & =f_{3}(s, t)+\frac{s-s^{2}}{i}+\frac{t-t^{2}}{j},
\end{aligned}
$$

where $f_{0}(s, t)=1, f_{1}(s, t)=s, f_{2}(s, t)=t$ and $f_{3}(s, t)=s^{2}+t^{2}$. Using these polynomials, we introduce the following positive linear operators on $C(S)$ :

$P_{i, j}(f ; s, t)=\left(1+\gamma_{i, j}(s, t)\right) B_{i, j}(f ; s, t), \quad(s, t) \in S=[0,1] \times[0,1]$ and $f \in C(S)$

where $\gamma_{i, j}(s, t)$ is given in Example 2.1. Now, take $A=C_{1}$, the Cesàro matrix. Since $\gamma_{i, j} \rightrightarrows \gamma=0\left(s t_{T}, \sigma\right)$, where

$$
\sigma(s, t)= \begin{cases}\frac{1}{s t}, & \text { if } \quad(s, t) \in(0,1] \times(0,1] \\ 0, & \text { if } s=0 \text { or } t=0\end{cases}
$$


Then, we conclude that

$$
P_{i, j}\left(f_{r}\right) \rightrightarrows f_{r}\left(s t_{T}, \sigma\right) \quad(r=0,1,2,3) .
$$

So by our main theorem, Theorem 3.2 , we immediately see that

$$
P_{i, j}(f) \rightrightarrows f \quad\left(s t_{T}, \sigma\right) \text { for } f \in C(S)
$$

However, since $\left(\gamma_{i, j}\right)$ is not triangular statistically uniformly convergent to $\gamma=0$ on the interval $S$, we can say that Theorem 3.1 does not work for our operators defined by (3.9).

\section{Rates of Triangular $A$-Statistical Relative Uniform Convergence}

In this section, using the notion of triangular $A$-statistical relative uniform convergence we study the rate of convergence of positive linear operators with the help of modulus of continuity.

Let $f \in C(S)$. Then the modulus of continuity of $f$, defined to be

$w(f ; \delta)=\sup \left\{|f(u, v)-f(s, t)|:(u, v),(s, t) \in S\right.$ and $\left.\sqrt{(u-s)^{2}+(v-t)^{2}} \leq \delta\right\}$

for $\delta>0$.

Then we hold the following result.

Theorem 4.1. Let $A=\left(a_{i, j}\right)$ be a nonnegative regular summability matrix. Let $\left(L_{i, j}\right)$ be a double sequence of positive linear operators acting from $C(S)$ into itself. Assume that the following conditions hold:

(a) $L_{i, j}\left(f_{0}\right) \rightrightarrows f_{0}\left(s t_{A}^{T}, \sigma_{0}\right)$,

(b) $w(f, \delta) \rightrightarrows 0\left(s t_{A}^{T}, \sigma_{1}\right)$, where $\delta:=\delta_{i, j}=\sqrt{\left\|L_{i, j}(\varphi)\right\|_{C(S)}}$, with $\varphi(u, v)=$ $(u-s)^{2}+(v-t)^{2}$.

Then we have, for all $f \in C(S)$,

$$
L_{i, j}(f) \rightrightarrows f \quad\left(s t_{A}^{T}, \sigma\right)
$$

where

$$
\sigma(s, t)=\max \left\{\left|\sigma_{0}(s, t)\right|,\left|\sigma_{1}(s, t)\right|,\left|\sigma_{0}(s, t) \sigma_{1}(s, t)\right|\right\},
$$

$\left|\sigma_{i}(s, t)\right|>0$ and $\sigma_{i}(s, t)$ is unbounded for $i=0,1$.

Proof. Let $f \in C(S)$ and $(s, t) \in S$ be fixed. Using linearity and positivity of $L_{i, j}$ we have, for any $(i, j) \in \mathbb{N}^{2}$ and $\delta>0$, 


$$
\begin{aligned}
& \left|L_{i, j}(f ; s, t)-f(s, t)\right| \\
= & \left|L_{i, j}(f(u, v)-f(s, t) ; s, t)-f(s, t)\left(L_{i, j}\left(f_{0} ; s, t\right)-f_{0}(s, t)\right)\right| \\
\leq & L_{i, j}(|f(u, v)-f(s, t)| ; s, t)+M\left|L_{i, j}\left(f_{0} ; s, t\right)-f_{0}(s, t)\right| \\
\leq & L_{i, j}\left(\left[1+\frac{\sqrt{(u-s)^{2}+(v-t)^{2}}}{\delta}\right] w(f ; \delta) ; s, t\right) \\
& +M\left|L_{i, j}\left(f_{0} ; s, t\right)-f_{0}(s, t)\right| \\
\leq & w(f ; \delta)\left|L_{i, j}\left(f_{0} ; s, t\right)-f_{0}(s, t)\right|+\frac{w(f ; \delta)}{\delta^{2}} L_{i, j}(\varphi ; s, t)+w(f ; \delta) \\
& +M\left|L_{i, j}\left(f_{0} ; s, t\right)-f_{0}(s, t)\right|,
\end{aligned}
$$

where $M=\|f\|_{C(S)}$. Taking the supremum over $(s, t) \in S$ in both sides of the above inequality, we obtain, for any $\delta>0$,

$$
\begin{aligned}
\left\|\frac{L_{i, j} f-f}{\sigma}\right\|_{C(S)} \leq & \frac{w\left(f, \delta_{i, j}\right)}{\left\|\sigma_{1}\right\|_{C(S)}}\left\|\frac{L_{i, j} f_{0}-f_{0}}{\sigma_{0}}\right\|_{C(S)}+\frac{w\left(f, \delta_{i, j}\right)}{\left\|\sigma_{1}\right\|_{C(S)} \delta^{2}}\left\|\frac{L_{i, j} \varphi}{\sigma_{1}}\right\|_{C(S)} \\
& +\frac{w\left(f, \delta_{i, j}\right)}{\left\|\sigma_{1}\right\|_{C(S)}}+M\left\|\frac{L_{i, j} f_{0}-f_{0}}{\sigma_{0}}\right\|_{C(S)} .
\end{aligned}
$$

Now, if take $\delta:=\delta_{i, j}=\sqrt{\left\|L_{i, j}(\varphi)\right\|}$, then we may write

$$
\left\|\frac{L_{i, j} f-f}{\sigma}\right\|_{C(S)} \leq \frac{w(f, \delta)}{\left\|\sigma_{1}\right\|}\left\|\frac{L_{i, j} f_{0}-f_{0}}{\sigma_{0}}\right\|_{C(S)}+2 \frac{w(f, \delta)}{\left\|\sigma_{1}\right\|}+M\left\|\frac{L_{i, j} f_{0}-f_{0}}{\sigma_{0}}\right\|_{C(S)}
$$

and hence,

$$
\left\|\frac{L_{i, j} f-f}{\sigma}\right\|_{C(S)} \leq K\left\{\frac{w(f, \delta)}{\left\|\sigma_{1}\right\|}\left\|\frac{L_{i, j} f_{0}-f_{0}}{\sigma_{0}}\right\|_{C(S)}+\frac{w(f, \delta)}{\left\|\sigma_{1}\right\|}+\left\|\frac{L_{i, j} f_{0}-f_{0}}{\sigma_{0}}\right\|_{C(S)}\right\}
$$

where $K=\max \{2, M\}$. For a given $r>0$, define the following sets: 


$$
\begin{aligned}
T: & =\left\{j \in \mathbb{N}: j \leq i,\left\|\frac{L_{i, j}(f)-f}{\sigma}\right\|_{C(S)} \geq r\right\}, \\
T_{1}: & =\left\{j \in \mathbb{N}: j \leq i, \frac{w(f, \delta)}{\left\|\sigma_{1}\right\|}\left\|\frac{L_{i, j} f_{0}-f_{0}}{\sigma_{0}}\right\|_{C(S)} \geq \frac{r}{3 K}\right\}, \\
T_{2}: & =\left\{j \in \mathbb{N}: j \leq i, \frac{w(f, \delta)}{\left\|\sigma_{1}\right\|} \geq \frac{r}{3 K}\right\}, \\
T_{3}: & =\left\{j \in \mathbb{N}: j \leq i,\left\|\frac{L_{i, j} f_{0}-f_{0}}{\sigma_{0}}\right\|_{C(S)} \geq \frac{r}{3 K}\right\} .
\end{aligned}
$$

It follows from (4.1) that

$$
T \subset T_{1} \cup T_{2} \cup T_{3}
$$

Also, define the sets:

$$
\begin{aligned}
& T_{4}:=\left\{j \in \mathbb{N}: j \leq i, \frac{w(f, \delta)}{\left\|\sigma_{1}\right\|} \geq \sqrt{\frac{r}{3 K}}\right\}, \\
& T_{5}:=\left\{j \in \mathbb{N}: j \leq i,\left\|\frac{L_{i, j} f_{0}-f_{0}}{\sigma_{0}}\right\|_{C(S)} \geq \sqrt{\frac{r}{3 K}}\right\} .
\end{aligned}
$$

Then observe that $T_{1} \subset T_{4} \cup T_{5}$. So we have $T \subset T_{2} \cup T_{3} \cup T_{4} \cup T_{5}$.

Therefore, using $(a)$ and $(b)$, the proof is complete.

\section{CONCLUSION}

If we take $A=C_{1}=\left(c_{i, j}\right)$, the Cesàro matrix defined by

$$
c_{i, j}:= \begin{cases}\frac{1}{i}, & \text { if } 1 \leq j \leq i \\ 0, & \text { otherwise }\end{cases}
$$

then triangular $A$-statistical relative uniform convergence reduces to the concept of triangular statistical relative convergence. Furthermore, if we take $A=C_{1}$ and the scale function by a non-zero constant, then triangular $A$-statistical relative uniform convergence reduces to the triangular statistical uniform convergence.

If one replaces the scale function by a non-zero constant, then the triangular $A$-statistical relative uniform convergence reduces to the triangular $A$-statistical uniform convergence.

\section{R E F E R E N C E S}

1. T. ACAR, S. A. Mohiuddine: statistical $(C, 1)(E, 1)$ summability and Korovkin's theorem. Filomat 30(2) (2016), 387-393. 
2. C. Bardaro, A. Boccuto, K. Demirci, I. Mantellini, S. Orhan: Triangular A-statistical approximation by double sequences of positive linear operators. Results Math. 68 (2015), 271-291.

3. C. Bardaro, A. Boccuto, K. Demirci, I. Mantellini, S. Orhan: Korovkintype theorems for modular $\Psi-A$-statistical convergence. J. Funct. Spaces Article ID 160401, 2015 (2015), p. 11.

4. C. BARdaro and I. Mantellini: Korovkin's theorem in modular spaces. Commentationes Math. 47 (2007), 239-253.

5. C. BARdaro and I. Mantellini: A Korovkin Theorem in multivariate modular function spaces. J. Funct. Spaces Appl. 7 (2009), 105-120.

6. C. Belen and S. A. Mohiuddine: Generalized weighted statistical convergence and application. Appl. Math. Comput. 219 (2013), 9821-9826.

7. E. W. Chitten: On the limit functions of sequences of continuous functions converging relatively uniformly. Trans. Amer. Math. Soc. 20 (1919), 179-184.

8. K. Demirci, F. DiRIK: A Korovkin type approximation theorem for double sequences of positive linear operators of two variables in A-statistical sense. Bull. Korean Math. Soc. 47(4) (2010) 825-837.

9. K. Demirci, B. Kolay: A-Statistical Relative Modular Convergence of Positive Linear Operators. Positivity 21 (2017), 847-863.

10. K. Demirci, S. Orhan: Statistical approximation by double sequences of positive linear operators on modular spaces. Positivity 19 (2015) 23-36.

11. K. Demirci, S. Orhan: Statistically relatively uniform convergence of positive linear operators. Results. Math. 69 (2016) 359-367.

12. K. Demirci, S. Orhan: Statistical relative approximation on modular spaces. Results. Math. 71 (2017) 1167-1184.

13. R. A. DeVore, G. G. Lorentz: Constructive Approximation ( Grund. Math. Wiss. 303). Springer Verlag, Berlin, 1993.

14. F. DiRIK, K. Demirci: Korovkin-type approximation theorem for functions of two variables in statistical sense. Turk. J. Math. 34 (2010) 73-83.

15. K. Donner: Korovkin Theorems in $L^{p}$ Spaces. J. Funct. Anal 42(1) (1981) 12-28.

16. S. M. Eisenberg: Korovkin's Theorem. Bull. Malays. Math. Soc. 2(2) (1979) 13-29.

17. H. FAST: Sur la Convergence Statistique. Colloq. Math. 2 (1951) 241-244.

18. P. Garrancho: A general Korovkin result under generalized convergence. Constr. Math. Anal. 2(2) (2019) 81-88.

19. A. D. Gadjiev, C. Orhan: Some approximation theorems via statistical convergence. Rocky Mountain J. Math. 32 (2002) 129-138.

20. U. KADAK, S. A. MoHIUDDINE: Generalized statistically almost convergence based on the difference operator which includes the $(p, q)$-gamma function and related approximation theorems. Results. Math. 73(1) (2018) 1-31.

21. P. P. Korovkin: Linear Operators and Approximation Theory. Hindustan Publ. Co., Delhi, 1960.

22. E. H. Moore: An Introduction to a Form of General Analysis. The New Hawen Mathematical Colloquim, Yale University Press, New Hawen, 1910. 
23. S. A. Mohiuddine: Statistical weighted A-summability with application to Korovkin's type approximation theorem. J. Inequal. Appl. 2016(1) (2016) 1-13.

24. S. A. Mohiuddine, B. A. S. AlAmri: Generalization of equi-statistical convergence via weighted lacunary sequence with associated Korovkin and Voronovskaya type approximation theorems. Rev. R. Acad. Cienc. Exactas Fis. Nat., Ser. A Mat., RACSAM 113(3) (2019) 1955-1973.

25. S. A. Mohiuddine, A. Asiri, B. HAzArika: Weighted statistical convergence through difference operator of sequences of fuzzy numbers with application to fuzzy approximation theorems. Int. J. Gen. Syst. 48(5) (2019) 492-506.

26. S. A. Mohiuddine, B. Hazarika, M. A. Alghamdi: Ideal relatively uniform convergence with Korovkin and Voronovskaya types approximation theorems. Filomat 33(14) (2019) 4549-4560.

27. F. MórICZ: Statistical Convergence of multiple sequences. Arch. Math. 81(1) (2003) 82-89.

28. A. Pringsheim: Zur theorie der zweifach unendlichen zahlenfolgen. Math. Ann. 53 (1900) 289-321.

29. G. M. Robison: Divergent double sequences and series. Amer. Math. Soc. Transl. 28 (1926) 50-57.

30. H. Steinhaus: Sur la Convergence Ordinaire et la Convergence Asymptotique. Colloq. Math. 2 (1951) 73-74.

31. P. ŞAhin, F. DiRIK: Statistical Relative Uniform Convergence of Double Sequence of Positive Linear Operators. Appl. Math. 17 (2017) 207-220.

32. T. O. Teoplitz: Über allgemeine lineare Mittelbildungen. Prace Math. Fiz. 22 (1911) 113-119.

33. B. Yilmaz, K. Demirci, S. Orhan: Relative Modular Convergence of Positive Linear Operators. Positivity 20 (2016), 565-577.

34. D. E. Wulbert: Convergence of Operators and Korovkin's theorem. J. Approx. Theory 1 (1968) 381-390. 